Association for Information Systems AIS Electronic Library (AISeL)

Wirtschaftsinformatik Proceedings 2005

Wirtschaftsinformatik

February 2005

\title{
Umsetzung eines ITIL-konformen IT-Service- Support auf der Grundlage von Web-Services
}

Gerrit Tamm

Universität St. Gallen

Rüdiger Zarnekow

Universität St. Gallen

Follow this and additional works at: http://aisel.aisnet.org/wi2005

\section{Recommended Citation}

Tamm, Gerrit and Zarnekow, Rüdiger, "Umsetzung eines ITIL-konformen IT-Service-Support auf der Grundlage von Web-Services" (2005). Wirtschaftsinformatik Proceedings 2005.34.

http://aisel.aisnet.org/wi2005/34

This material is brought to you by the Wirtschaftsinformatik at AIS Electronic Library (AISeL). It has been accepted for inclusion in Wirtschaftsinformatik Proceedings 2005 by an authorized administrator of AIS Electronic Library (AISeL). For more information, please contact elibrary@aisnet.org. 
In: Ferstl, Otto K, u.a. (Hg) 2005. Wirtschaftsinformatik 2005: eEconomy, eGovernment, eSociety; 7. Internationale Tagung Wirtschaftsinformatik 2005. Heidelberg: Physica-Verlag

ISBN: 3-7908-1574-8

(C) Physica-Verlag Heidelberg 2005 


\title{
Umsetzung eines ITIL-konformen IT-Service- Support auf der Grundlage von Web-Services
}

\author{
Gerrit Tamm, Rüdiger Zarnekow \\ Universität St. Gallen
}

\begin{abstract}
Zusammenfassung: Die Umsetzung von IT-Service-Support-Prozessen auf der Basis des Referenzmodells IT Infrastructure Library (ITIL) geschieht in vielen Unternehmen derzeit mit hoher Priorität. Obwohl zur Unterstützung der SupportProzesse eine Vielzahl von Softwarelösungen zur Verfügung stehen, basieren diese heute nicht auf Service-orientierten-Architekturen. Die Vorteile einer Serviceorientierten-Architektur werden daher im Rahmen des IT-Service-Supports nicht genutzt. Der Beitrag zeigt am Beispiel eines zentralen ITIL-Support-Prozesses, dem Incident-Management, wie dieser technisch auf der Basis von Web-Services umgesetzt werden kann. Die Grundlage bilden sechs Fallstudien, in denen die Gestaltung ITIL-basierter Support-Prozesse untersucht wurde. Aufbauend auf den Fallstudien wird ein generischer Incident-Management-Prozess definiert und mit Hilfe von Web-Services umgesetzt. Dabei kommen sowohl atomare als auch aggregierte Web-Services zum Einsatz. Für ein konkretes Service-Anliegen wird der Prozessablauf grafisch beschrieben. Die Umsetzung erfolgt exemplarisch unter Zuhilfenahme der Web Services Description Language (WSDL).
\end{abstract}

Schlüsselworte: IT-Service-Management, ITIL, Web-Services, Service-orientierteArchitekturen

\section{Einleitung und Motivation}

IT-Organisationen in Unternehmen durchlaufen einen Transformationsprozess von einem reinen Lieferanten für Informationstechnik hin zu einem Dienstleistungserbringer (Service Provider) für das gesamte Unternehmen [Zarn04]. Im Zentrum dieser Dienstleistungsorientierung steht eine verstärkte Kunden- und Prozessausrichtung der IT-Organisation, d. h. eine konsequente Ausrichtung aller ITProzesse an den Kundenanforderungen [BöKr04]. Nicht zuletzt aus diesem Grund ist in jüngster Zeit ein verstärktes Interesse an Best-Practices und Referenzmodellen zur Gestaltung Service-orientierter IT-Managementprozesse zu beobachten [HoHu03]. Insbesondere die IT Infrastructure Library (ITIL) hat sich diesbezüglich zu einem de-facto Standard entwickelt [HoZa04]. 
Ein zentrales Element Service-orientierter IT-Managementprozesse bildet der ITService-Support, d. h. die Unterstützung von Endkunden und Nutzern. Die Wahrnehmung der IT-Organisation im Unternehmen und die Zufriedenheit der Nutzer mit den IT-Services hängt stark von der Qualität des Service-Supports ab. Das häufig schlechte Image der IT-Organisation im Unternehmen und Vorwürfe hinsichtlich einer fehlenden Kundenorientierung, geringen Transparenz und mangelhaften Qualität der IT-Services [HoHo98] sind nicht zuletzt bedingt durch eine unzureichende Gestaltung des IT-Service-Supports.

Organisatorisch wird der IT-Service-Support in der Praxis durch einen Help- oder Service-Desk erbracht. Die erforderlichen Support-Prozesse sind in der Regel sehr strukturiert aufgebaut und orientieren sich an den in der ITIL vorgeschlagenen Best-Practice-Prozessen (siehe Abschnitt 2.1). Für die Abwicklung der SupportProzesse stehen eine Reihe von Softwarewerkzeugen und -lösungen zur Verfügung, die die Service-Desk-Mitarbeiter bei ihrer Arbeit unterstützen [LeDi04]. Keine der heute verfügbaren Lösungen basiert jedoch technologisch auf einer Service-orientierten-Architektur. Vorteile, etwa durch den Einsatz von Web-Services, bleiben daher im Bereich des IT-Service-Support ungenutzt. Hierzu zählen beispielsweise eine flexible Servicearchitektur für heterogene Endgeräte, ein nutzerspezifischer Mensch-Maschine-Support und eine automatische Selektion und Bereitstellung von IT-Service-Supportfunktionen.

Vor diesem Hintergrund untersucht der Beitrag die Möglichkeiten der Umsetzung eines ITIL-konformen IT-Service-Support auf der Basis von Web-Services. Zunächst findet in Kapitel 2 eine kurze Beschreibung der Grundlagen der ITIL und des Einsatzes von Web-Services statt. Daran anschliessend werden am Beispiel eines zentralen ITIL-Support-Prozesses, dem Incident-Management, die Möglichkeiten einer Web-Service-basierten Implementierung aufgezeigt. Kapitel 3 beschreibt ein generisches Umsetzungsmodell. Kapitel 4 stellt die konkrete Implementierung des Modells exemplarisch für ein Service-Anliegen vor. Kapitel 5 zeigt die zentralen Erkenntnisse auf, die bei der Implementierung in der Praxis gesammelt werden konnten. Den Abschluss bildet Kapitel 6 mit einem zusammenfassenden Fazit.

Die methodische Grundlage des beschriebenen Umsetzungskonzeptes bilden sechs Fallstudien, in denen die Gestaltung ITIL-basierter Service-Support-Prozesse bei 3M Deutschland, BASF IT Services, DaimlerChrysler, Kreditanstalt für Wiederaufbau, Stadt Köln und T-Mobile untersucht und ausgewertet wurde. Auf der Grundlage der sechs analysierten, unternehmensindividuellen IT-Support-Prozesse wurde ein generisches Modell entwickelt, das die Basis für das Umsetzungskonzept bildet. 


\section{Grundlagen}

\subsection{IT Infrastructure Library (ITIL)}

Seit Ende der 80er Jahre entwickelt die Central Computer and Telecommunications Agency (CCTA) der britischen Regierung (mittlerweile Bestandteil des Office of Government Commerce) in Zusammenarbeit mit IT-Spezialisten, Rechenzentrumsbetreibern und Beratern eine prozessorientierte Sammlung von Best Practices für die Planung, Überwachung und Steuerung von IT-Services [OGC00]. Im Mittelpunkt der ITIL steht die konsequente Serviceorientierung von IT-Dienstleistern. IT-Services müssen ausgehend von den Kundenanforderungen definiert und die internen Prozesse eines IT-Dienstleisters an den Kundenanforderungen ausgerichtet werden. In jüngster Zeit hat sich die ITIL zu einem internationalen de-facto Standard für IT-Dienstleister entwickelt und bildet als herstellerunabhängige Sammlung von Best Practices die Grundlage für das international tätige ITService-Management-Forum (ITSMF) mit mittlerweile über 2.000 Partnerunternehmen [ITSM04].

Die ITIL besteht im Kern aus fünf Bausteinen, die in Form einzelner Buchbände veröffentlicht sind. Der Band "Business-Perspective" umfasst die strategischen Elemente des IT-Service-Managements, wie z. B. das IT-Alignment oder Relationship-Management. "Service-Delivery" beschäftigt sich mit der Planung, Überwachung und Steuerung von IT-Services. Innerhalb des "Service-Support" werden die Umsetzung der Service-Prozesse sowie der Nutzer-Support behandelt. Das Management von Applikationen über ihren gesamten Lebenszyklus hinweg ist Betrachtungsgegenstand des "Application-Management". Das "ICT-InfrastructureManagement" schliesslich beschreibt die Prozesse zur Planung, Umsetzung, Betrieb und technischen Support der IT-Infrastruktur.

Praktische Bedeutung kommt vor allem den Bausteinen "Service-Support" und "Service-Delivery" zu. Da in diesem Beitrag der Service-Support im Mittelpunkt steht, wird dieser im Folgenden kurz näher beschrieben. Die ITIL gliedert den Service-Support in die Teilbereiche Incident-Management, Problem-Management, Change-Management, Release-Management und Configuration-Management (siehe Abbildung 1). 


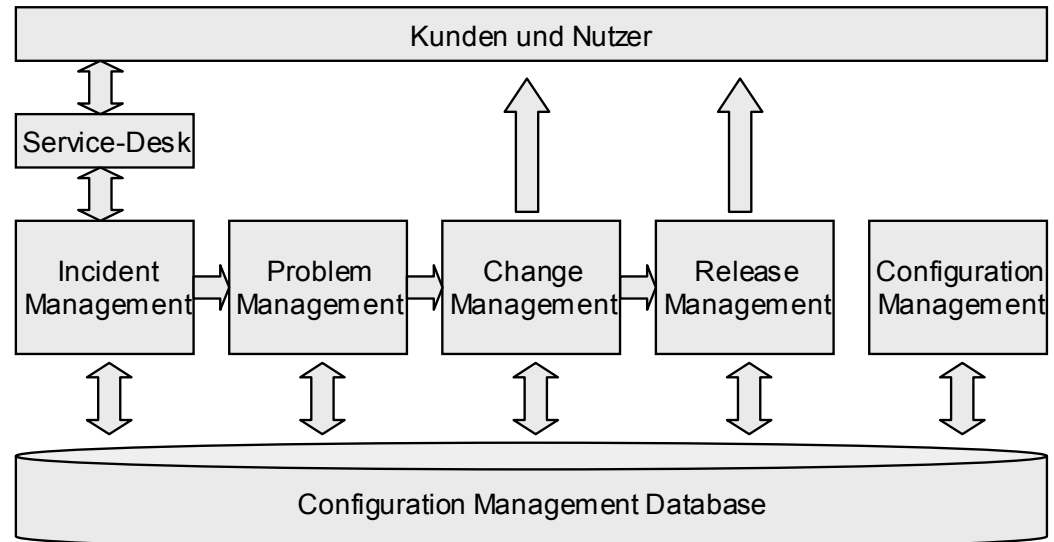

Abbildung 1: Bausteine des Service-Support gemäss ITIL (in Anlehnung an [OGC00])

Die Schnittstelle zu den Kunden und Nutzern wird organisatorisch durch ein Service-Desk gebildet. Im Service-Desk läuft der Incident-Management-Prozess ab. Die Aufgaben des Incident-Managements umfassen die Aufnahme und Bearbeitung von Support-Anliegen, sowie deren Koordination, Überwachung und Kommunikation. Ziel des Problem-Managements ist es, die für das Auftreten von Störungen verantwortlichen Probleme reaktiv und proaktiv zu identifizieren und zu beheben. Die Problembehebung erfordert in der Regel Änderungen an der ITInfrastruktur. Damit der Änderungsprozess nicht unkontrolliert abläuft, ist es Aufgabe des Change-Managements, standardisierte Prozesse und Kontrollprozeduren für IT-bezogene Änderungen bereitzustellen. Das Release-Management umfasst die Sicherstellung einer anforderungsgerechten Planung, Entwicklung und Implementierung von Software- oder Hardware-Releases. Das ConfigurationManagement dient der Verwaltung und Kontrolle der IT-Infrastruktur und der ITServices. Es ist für die Pflege der Configuration-Management-Database (CMDB) zuständig, in der sämtliche Infrastruktur- und Servicekomponenten, sowie deren Beziehungen untereinander, abgebildet sind. Die CMDB steht den anderen Support-Prozessen als Informationsbasis zur Verfügung.

\subsection{Web-Services}

Web-Services und Service-orientierte-Architekturen nehmen in der wissenschaftlichen und praxisorientierten Fachliteratur einen zunehmend hohen Stellenwert ein. Innerhalb kürzester Zeit haben sich Web-Service-Standards weltweit etabliert. Führende Softwareanbieter nutzen Web-Service-Technologien als Basis für die Entwicklung neuer Generationen ihrer klassischen, monolithischen Softwaresysteme (z. B. ERP-Systeme) und passen diese für eine Nutzung auf Basis von Inter- 
netstandards an. Die steigende Vernetzung der Wertschöpfungssysteme und die damit verbundenen hohen Anforderungen an Flexibilität und Transparenz motiviert die zunehmende Bereitstellung von Web-Services. Standardisierte Schnittstellen und Auszeichnungssprachen der Web-Services auf Basis von XML ermöglichen die flexible Vernetzung zwischen Web-Services. Die Vision einer vernetzten Welt, in der Nutzer ihre Bedürfnisse abstrakt spezifizieren können und diese dann über komplexe, selbst-konfigurierende Web-Services (z. B. Aggregatoren für webbasierte Dienste) gedeckt werden, impliziert dass einzelne Web-Services automatisch andere Web-Services finden und aufrufen können. Auch wenn die technologischen Standards im Bereich Service-orientierter-Architekturen breite Akzeptanz erzielen, gibt es noch eine Vielzahl von ökonomischen und organisatorischen Barrieren, welche die Entwicklung des gesamten Marktes für Web-Services hemmen. Neben offenen Fragen im Bereich Sicherheit, Preisbildung und Performanz haben wir vor allem das mangelnde Vertrauen in Web-Services als eine wesentliche Barriere identifiziert [TaWu03].

Im Folgenden werden diejenigen Web-Service-Standards, die für das später beschriebene Umsetzungskonzept relevant sind, kurz vorgestellt. Darüber hinaus werden Web-Service-Technologien, mit Hilfe derer Web-Services aggregiert werden können, beschrieben. Der Aggregationsmechanismus bildet einen zentralen Baustein des Umsetzungskonzeptes.

\section{Etablierte Standards für Web-Services}

Web-Services beschreiben aus technologischer Sicht kein völlig neues Konzept. Der Erfolg der Web-Service-Technologie liegt vor allem darin begründet, dass führende Softwareunternehmen Web-Service-Standards gemeinsam entwickeln und vermarkten. Darüber hinaus kann im Vergleich zu anderen Ansätzen eine deutliche Reduktion der Komplexität erzielt werden. Die Ursprünge der WebService-Technologie liegen in Bestrebungen einzelner Unternehmen oder Konsortien, einen Standard für eine integrierte, verteilte Softwarearchitektur zu etablieren. Eine Kernfunktionalität der Unix-Betriebssysteme, die „Remote procedure calls (RPCs)“, Microsofts „Distributed Component Object Model (DCOM)“ und die „Common Object Request Broker Architecture (CORBA)“ sind einige Beispiele für technologische Standards mit vergleichbaren Zielen. Gisolfi [Giso01] beschreibt im Detail die Unterschiede zwischen Web-Services, DCOM and CORBA. Mögliche Probleme bei der Kombination von komponentenbasierter Middleware (CORBA) mit Web-Service-Technologien beschreibt Stal [Stal02].

Web-Services sind modulare, selbstbeschreibende Softwarekomponenten und bilden die Grundlage für eine flexible Service-orientierte-Architektur, welche auf offenen und allgemein akzeptierten Standards (z. B. XML, HTTP) beruht. Aufgrund der Standardisierung können Web-Services zu komplexen Architekturen zusammengesetzt werden. Hierbei können einzelne Web-Services andere WebServices finden und aufrufen. Diese Eigenschaft macht sie besonders geeignet da- 
für, etablierte Referenzmodelle, wie z. B. ITIL, abzubilden. Sowohl einzelne als auch aggregierte Web-Services basieren auf folgenden grundlegenden WebService-Standards.

Die "Extensible Markup Language (XML)“ bildet die Basis für alle Web-ServiceAuszeichnungsstandards. Das Datenformat des Nachrichtenaustauschs und die Auszeichnung der Schnittstelle zwischen Web-Services basiert auf XML.

Das „Simple Object Access Protocol (SOAP)” [W3C00] ist das Protokoll für den Nachrichtenaustausch zwischen Web-Services auf Basis von XML. SOAP 1.2 [W3C03] wird seit Juni 2003 als Standard des World Wide Web Konsortiums empfohlen.

Die "Web-Service Description Language (WSDL)" [W3C01] ist die Auszeichnungssprache für Web-Services. Bevor SOAP die Kommunikation zwischen mehreren Web-Services ermöglicht, ist eine Spezifizierung der Kommunikationsschnittstellen, Zugangsspezifikationen und Lokalitäten der einzelnen WebServices mit Hilfe von WSDL notwendig. Innerhalb eines WSDL-Dokuments werden die einzelnen Funktionen eines Web-Services und deren Input- und Outputparameter beschrieben. Das Element „types“ deklariert die Datentypen der Nachrichten. Das Element „,message“ beschreibt die Input- und Outputparameter einer Transaktion (Operation) eines Web-Services. Mit dem Standard WSDL 2.0 können neben den klassischen Transaktionen "unidirektional" (One-way), "bidirektional" (Request-Response), "Warten auf Antwort" (Solicit-response) und "Benachrichtigung" (Notification) auch individuelle Transaktionen definiert werden. Mit Hilfe einer Service-orientierten-Architektur ist es daher auch möglich, auf eine Service-Anfrage, z. B. eine IT-Service-Support-Anfrage, eine Auswahl möglicher IT-Service-Support-Services bereitzustellen. Das Element „portType“ ist das wichtigste Element eines WSDL-Dokumentes und definiert die einzelnen Transaktionen eines Web-Services und die dafür notwendigen Nachrichten (Input und Output). Transaktionen eines Web-Services sind mit Funktionen einer klassischen Software vergleichbar. Ein so genanntes „binding element” spezifiziert die Bindung eines Web-Services an ein spezielles Protokoll, die Abfolge spezifischer Ausführungen, die Dekodierung der SOAP-Nachrichten und schließlich den Standort des Web-Services. WSDL ermöglicht es Anbietern von webbasierten Diensten, spezifische Transaktionen (operations) als Bedingung zum Aufruf eines elementaren Web-Services oder einer Gruppe von Web-Services aufzustellen. Ein standardisierter Web-Service, z. B. für eine Zahlungsfunktion, kann vor Aufruf des angeforderten Web-Services als Vorbedingung definiert werden. Nicht spezifischen Web-Services, wie z. B. einem einfachen IT-Support-Interface-Service, stehen somit eine Vielzahl von spezifischen Web-Services, z. B. IT-SupportProblemlösungs-Services einer spezifischen Branche, als vor- und nachgelagerte Web-Services zur Verfügung.

Die „Universal Description, Discovery and Integration (UDDI)“ [OASI03] ist die zentrale Registrierungsinitiative für Web-Services. Alle grundlegenden Standards 
für Web-Services werden kontinuierlich durch die Standardisierungsinstitutionen "World Wide Web Consortium (W3C)" und "Organization for the Advancement of Structured Information Standards (OASIS)"11 weiterentwickelt und überprüft.

\section{Service-orientierte-Architekturen}

Interoperabilität und Interaktion zwischen verteilten Web-Services auf Basis standardisierter Technologien bildet die Grundlage für eine Service-orientierteArchitektur (SOA). Innerhalb einer SOA bietet ein Web-Service selbstbeschreibende Funktionalität über standardisierte Schnittstellen an. Die Funktion des WebServices kann für unterschiedliche Kontexte berücksichtigt werden und ermöglicht damit die Wiederverwendung und Mehrfachverwendung eines nicht spezifischen Web-Services. Eine SOA ist eine aggregierte, hochflexible Architektur aus mehreren Web-Services. Stevens [Stev02] beschreibt im Detail die Vorteile einer SOA, insbesondere im Hinblick auf den hohen Flexibilitätsgrad. Ein wesentliches Merkmal der SOA liegt im Vergleich zur objektorientierten Programmierung in der Trennung von Funktionen und Daten. He [He03] vergleicht das Konzept der objektorientierten Programmierung mit dem Konzept der SOA. Als Fazit beschreibt er die Vorteile der Trennung von Funktionen und Daten für digitale Güter im SOA-Konzept. Innerhalb einer SOA lassen sich mindestens drei verschiedene Rollen klassifizieren [Giso01]: Service-Nachfrager (Service Requestor), ServiceAnbieter (Service Provider) und Service-Marktplatz (Service Repository). Den Zusammenhang zwischen den einzelnen Rollen veranschaulicht Abbildung 2.

Der Service-Nachfrager kann innerhalb des Service-Registers eines Marktplatzes nach passenden Angeboten suchen. Der Service-Anbieter beschreibt seine WebServices mit Hilfe des Service-Registers und nutzt dieses auch für die Vermarktung seiner Dienste. Nachdem der Service-Nachfrager einen passenden Service im Service-Register gefunden hat, kann er den Web-Service beim Service-Anbieter aufrufen.

\footnotetext{
http://www.w3.org, http://www.oasis-open.org
} 


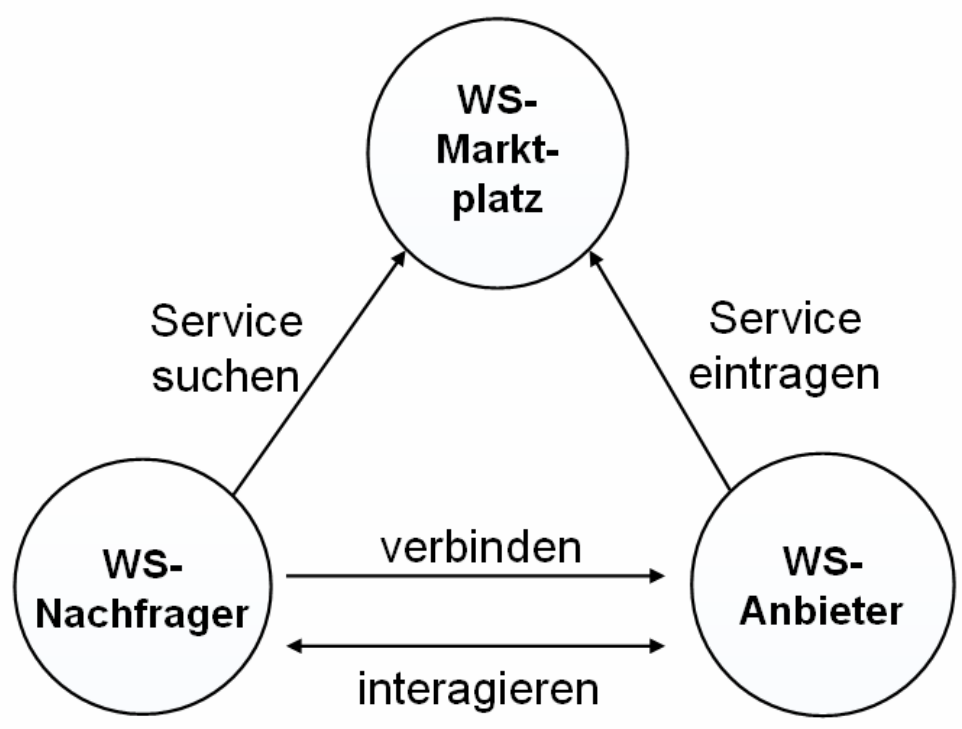

Abbildung 2: Die Rollen einer SOA

Eine besondere Rolle in einer SOA stellt der Aggregator-Web-Service dar. Ein Aggregator-Web-Service bündelt einzelne Web-Services zu einem komplexen Web-Service und kann selbst als Web-Service aufgerufen werden. Innerhalb des Aggregator-Web-Services werden die nachfragerspezifischen Anforderungen mit Hilfe einer geeigneten Matchingfunktion in standardisierte Anforderungen an eine Vielzahl einzelner Web-Services transformiert. Für Service-Nachfrager kann der Aggregator-Web-Service eine Vielzahl von Service-Angeboten identifizieren, bewerten, aggregieren und schließlich ausführen. Abbildung 3 veranschaulicht vereinfachend die Rolle eines Service-Aggregators.

Atomare und aggregierte Web-Services bilden die Basisbausteine einer SOA. Atomare Web-Services bieten definierte, selbstbeschreibende Funktionalität, welche unabhängig von anderen Web-Services ist. Aggregierte Web-Services rufen atomare Web-Services oder bereits aggregierte Web-Services auf und sind daher abhängig von den ihnen zugrunde liegenden atomaren Web-Services. 


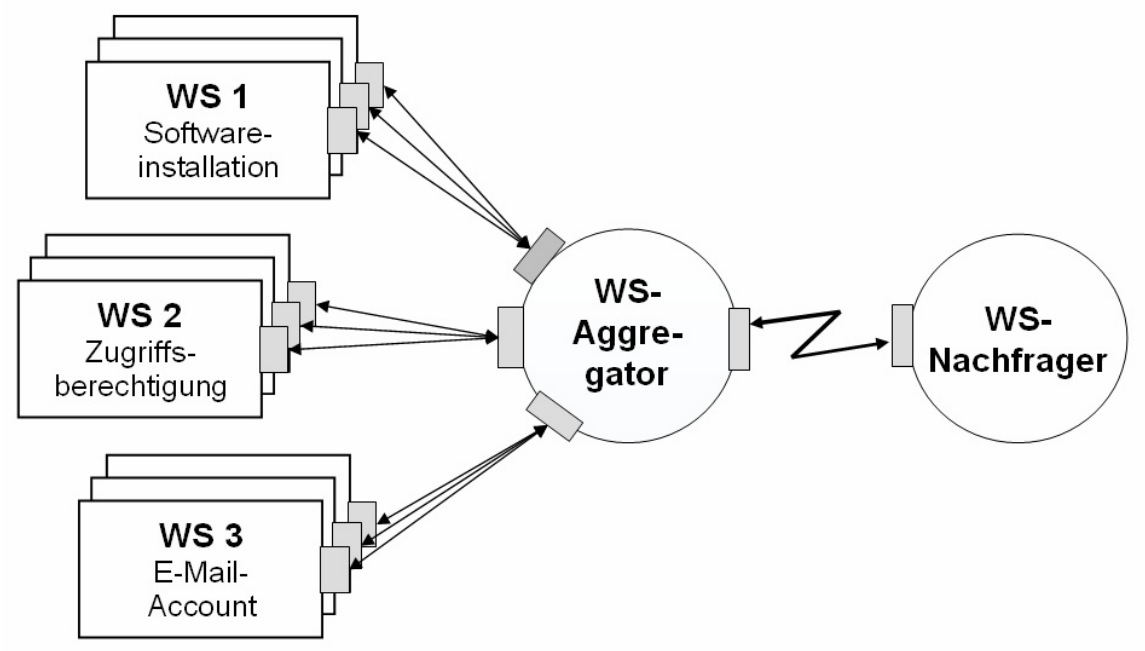

Abbildung 3: Der Web-Service-Aggregator

Für die Realisierung von komplexen Referenzmodellen, wie z. B. ITIL, in einer SOA muss ein definierter Workflow zwischen den teilnehmenden Web-Services definiert werden. Der Workflow muss die Anforderungen für die vor- und nachgelagerten Aufgaben, die Abfolge der Web-Service-Ausführung, eine Übersicht der verantwortlichen Web-Services pro Aufgabe und die Vorgaben bezüglich der Ausführungszeit beinhalten. Mit Hilfe geeigneter Monitoringsysteme können SOA kontinuierlich überwacht und gesteuert werden [QuWi03]. Dies ist z. B. dann erforderlich, wenn einzelne atomare Web-Services erst ausgeführt werden sollen, nachdem vorgelagerte Web-Services vollständig ausgeführt worden sind. Der Workflow innerhalb einer SOA kann mit Hilfe der "Business Process Execution Language for Web-Services (BPEL4WS)" [BPEL03] realisiert werden. Die Standardisierung von BPEL4WS erfolgte im April 2003 durch OASIS. In dem Standard wurden zwei Konzepte integriert: IBM WSFL (Web-Service Flow Language) und Microsoft's XLANG. Mit BEA, SAP und Siebel waren drei weitere führende Softwareanbieter an der Entwicklung von BPEL4WS beteiligt. Mittels BPEL4WS können vernetzte Geschäftsprozesse als SOA abgebildet werden. 


\section{Umsetzung einer Service-Orientierten-Architektur für das Incident-Management}

\subsection{Grundmodell des Incident-Management-Prozesses gemäss ITIL}

Im Folgenden soll am Beispiel des Incident-Management-Prozesses beschrieben werden, wie ein ITIL-konformer Referenzprozess technologisch auf der Basis einer SOA umgesetzt werden kann. Das Incident-Management wurde ausgewählt, weil es innerhalb des IT-Service-Supports die unmittelbare Schnittstelle zum Nutzer bildet und aus diesem Grund die Vorteile einer SOA in hohem Masse zum Tragen kommen.

Um ein generisches Modell eines Incident-Management-Prozesses entwickeln zu können, wurden in sechs Fallstudien ITIL-konforme Incident-ManagementProzesse in Unternehmen analysiert. Demnach besteht das Incident-Management typischerweise aus den in Abbildung 4 dargestellten Teilprozessen.

Ausgangspunkt des Prozesses ist der Nutzer mit einem konkreten SupportAnliegen. In den betrachteten Unternehmen existiert ein Service-Desk, an den sich der Nutzer telefonisch oder elektronisch, z. B. per E-Mail, wenden kann. Das Support-Anliegen des Nutzers wird als Incident erfasst und im Anschluss klassifiziert. Auf der obersten Klassifikationsebene unterscheidet die ITIL zwischen Service-Anliegen und Störungen. Typische Service-Anliegen innerhalb eines Unternehmens sind beispielsweise die Einrichtung eines E-Mail-Accounts, die Einrichtung eines neuen Arbeitsplatzssystems, die Einrichtung eines neuen Druckers oder die Vergabe eines Passwortes. Für diese Service-Anliegen existieren vorgegebene Prozesse, die in der Regel unmittelbar im Service-Desk bearbeitet werden können. Handelt es sich bei dem Support-Anliegen des Nutzers hingegen um eine Störungsmeldung, ist diese genauer zu analysieren. Üblicherweise werden die Störungen in unterschiedliche Störungstypen, z. B. Netzwerkstörungen, Störungen in Anwendungsprogrammen oder Hardware-Störungen, unterteilt. Durch den Zugriff auf eine Problem-Datenbank, in der alle bekannten Störungen (Known-Errors) und die dazugehörigen Lösungen gespeichert sind, kann festgestellt werden, ob es sich um eine bereits bekannte Störung handelt, für die ein Lösungsvorgehen vorliegt. Ist dies der Fall, kann die Störung im Rahmen des Incident-Managements behoben werden. Ist die Störung hingegen unbekannt, wird sie an das ProblemManagement übergeben, da innerhalb des Incident-Managements keine weitergehende Problemanalyse und -lösung stattfindet. Der Problem-Management-Prozess ist ebenfalls in der Abbildung dargestellt, wird jedoch an dieser Stelle nicht näher beschrieben. Bei einer unbekannten Störung erarbeitet das Incident-Management einen Workaround, der es dem Nutzer ermöglicht, bis zur Behebung der Störung seine Arbeit weiter zu verrichten. Ist beispielsweise ein Drucker gestört, so kann 
das Incident-Management im Sinne eines Workarounds den Nutzer mit einem anderen Drucker verbinden, solange bis die eigentliche Störung behoben ist. Der Incident-Management-Prozess endet mit dem Schliessen des Incidents und einer Information des Nutzers.

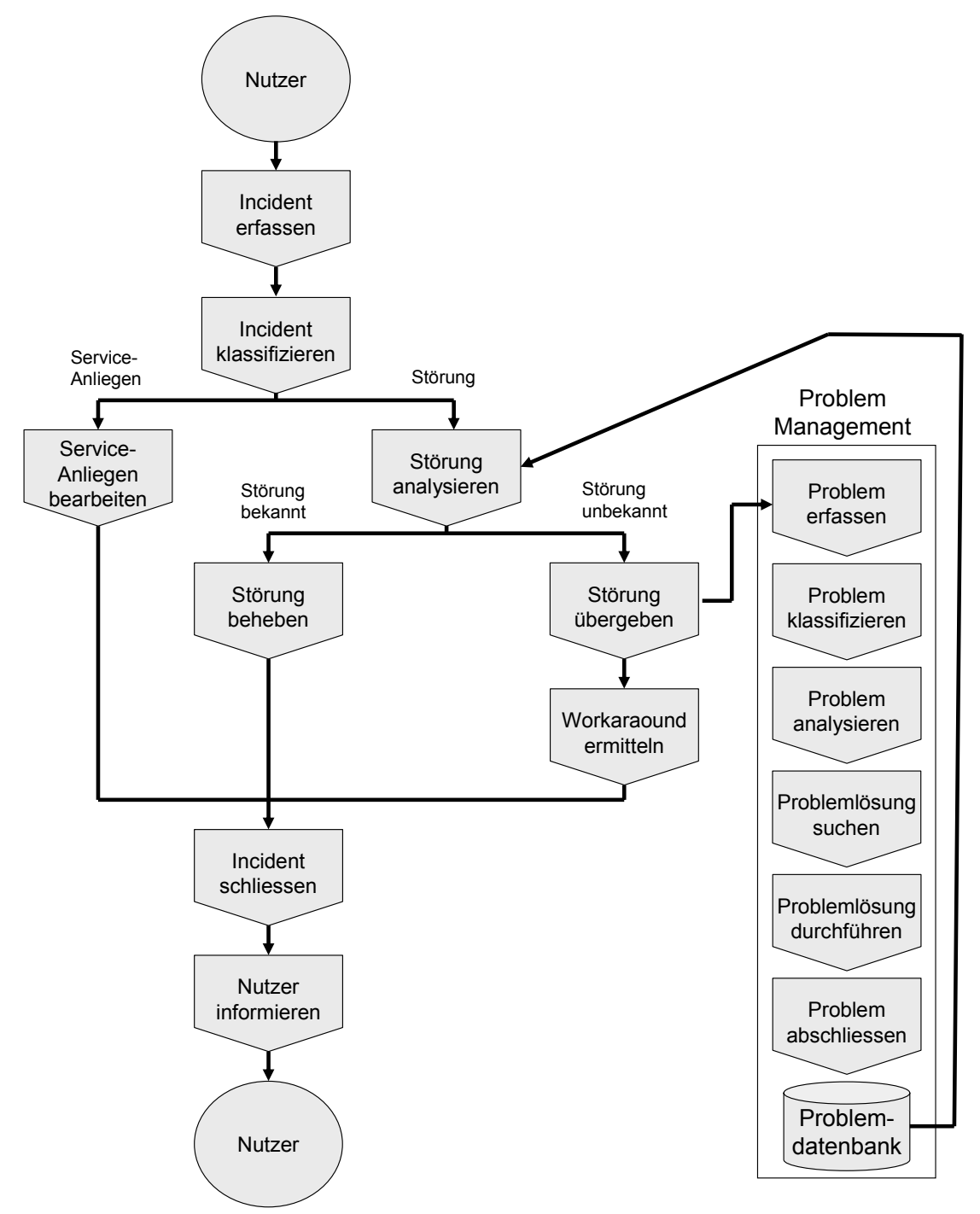

Abbildung 4: Generischer ITIL-konformer Incident-Management-Prozess

Das Incident-Management wird bei allen untersuchten Unternehmen durch Softwarewerkzeuge unterstützt. Bei den Werkzeugen handelt es sich sowohl um spe- 
zielle Lösungen für den Service-Desk, als auch um Komplettlösungen für sämtliche Bereiche des IT-Service-Managements. In den Werkzeugen ist der beschriebene Prozess in Form eines Workflows abgebildet. Die Lösungen basieren systemseitig auf einer Client/Server-Architektur. Die Mitarbeiter im Service-Desk greifen über einen Client, z. T. auch einen Web-Client, auf den Systemserver zu, der eine zentrale Configuration-Management-Database als Datenbasis nutzt [LeDi04]. Eine direkte Schnittstelle zum Endnutzer, um diesem beispielsweise die eigenständige Eingabe von Incidents zu ermöglichen, ist systemseitig in der Regel nicht realisiert.

\subsection{Umsetzung des Grundmodells auf der Basis von Web- Service-Standards}

Unter Berücksichtigung der Web-Service-Standards, im Speziellen des BPEL4WS-Standards, kann der beschriebene ITIL-konforme Incident-Management-Prozess als SOA wie folgt gestaltet werden (siehe Abbildung 5).

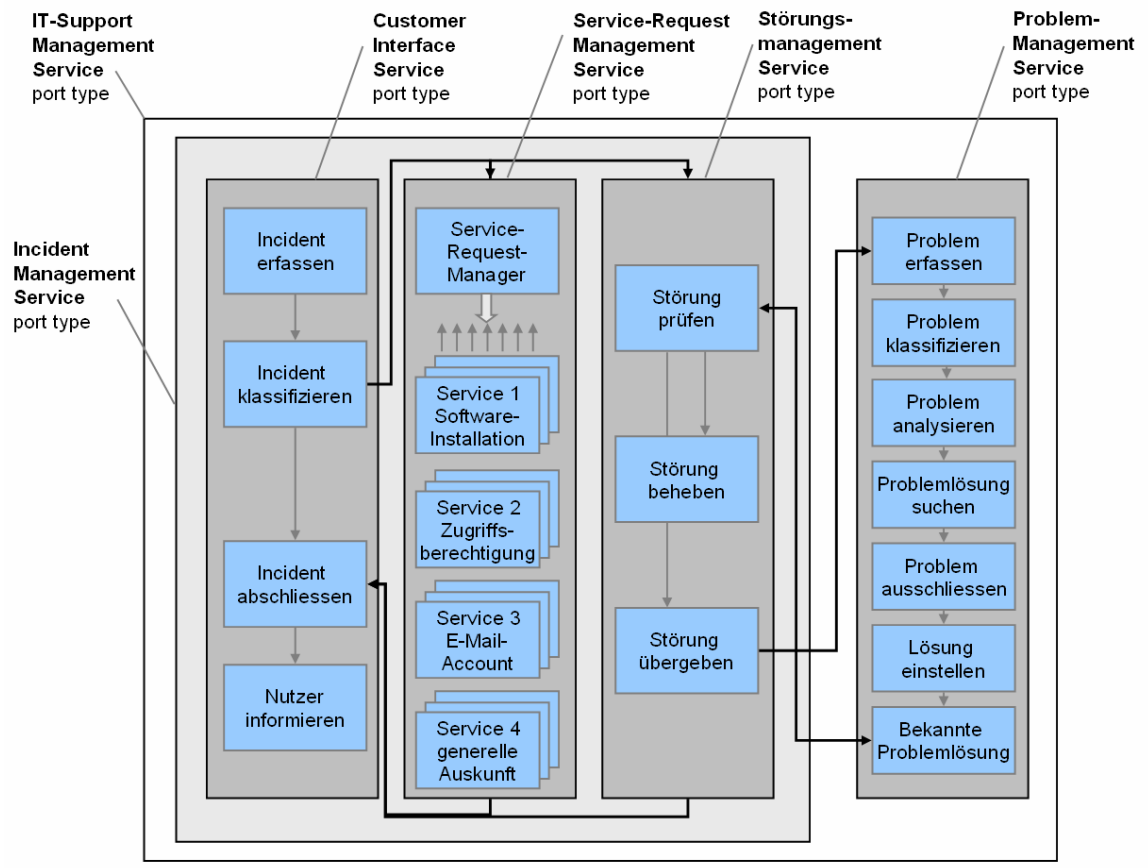

Abbildung 5: IT-Support-Management-Service als SOA

Als aggregierter Web-Service setzt sich der "IT-Support-Management-Service" aus den aggregierten Web-Services "Incident-Management-Service" und "Problem-Management-Service" zusammen. Innerhalb des "Incident-Management- 
Service" werden die Web-Services mit den Funktionen für die Kundenschnittstelle zum "Customer-Interface-Service" aggregiert. Dieser setzt sich aus den atomaren Web-Services „Incident erfassen“, „Incident klassifizieren“, „Incident abschliessen“ und „Nutzer informieren“ zusammen. Sofern der Web-Service „Incident klassifizieren“ die Anfrage des Nachfragers als Service-Anliegen klassifiziert, erfolgt eine Transaktionen „Request-Respond“ an den aggregierten Web-Service "Service-Request-Management-Service". Mit Hilfe der Transaktion „request multiple responses" können diejenigen relevanten und verfügbaren atomaren Web-Services identifiziert werden, welche dem spezifischen Service-Anliegen des Kunden am ehesten entsprechen. Der "Service-Request-Manager" wählt die passenden atomaren Web-Services aus und sendet eine „Notification“-Transaktion an den Web-Service „Incident abschliessen“. Der Web-Service-Nachfrager wird schließlich über den Web-Service „Nutzer informieren“ über die Bereitstellung der angeforderten Web-Services und deren Funktionen informiert und kann mit diesen interagieren.

Sofern der atomare Web-Service „Incident klassifizieren“ eine Störung identifiziert, erfolgt eine Transaktion ,request-respond“ an den aggregierten Web-Service "Störungs-Management-Service". Der atomare Web-Service „Bekannte Problemlösungen" sucht innerhalb einer Datenbank mit bekannten Problemlösungen nach verfügbaren Lösungen zur Behebung der Störung. Sofern die Behebung der Störung z. B. an bestimmte Zeitrestriktionen auf Nachfragerseite gebunden oder nicht gebunden ist, kann die Transaktion als „Solicit-response“ oder „request-multiple response“ realisiert werden. Sofern der Web-Service „Störung prüfen“ eine bekannte Problemlösung identifiziert, kann diese als „One-way“ Transaktion an den aggregierten Web-Service "Customer-Interface-Service" realisiert werden. Der Web-Service-Nachfrager wird in diesem Fall durch den Web-Service „Nutzer informieren“ über die spezifische Problemlösung informiert. Sofern jedoch der Web-Service „Störungen prüfen“ innerhalb des geforderten Zeitraums keine Antwort von dem Web-Service „Bekannte Problemlösungen“ erhält und somit keine bekannte Problemlösung bereitgestellt werden kann, wird die Störung durch den Web-Service „Störung übergeben“ an den aggregierten Web-Service "ProblemManagement-Service" übergeben. Der aggregierte Web-Service "StörungsManagement-Service" sendet eine „Notification“, z. B. mit einem Hinweis auf die erwartete Bearbeitungszeit, an den atomaren Web-Service „Incident abschließen“. Über den atomaren Web-Service „Nutzer informieren“ wird die „Notification“ an den Web-Service-Nachfrager gesendet.

Mit Hilfe der Web-Service-Standards, insbesondere mit BPEL4WS, kann das ITIL-Referenzmodell als Service-orientierte-Architektur abgebildet werden. Spezifische und nicht-spezifische, atomare und aggregierte Web-Services können identifiziert werden, und die Interaktion zwischen den Web-Services kann über geeignete Transaktionen erfolgen. Wie die detaillierten XML-Elemente eines ITILWeb-Services ausgeprägt sind, wird im nächsten Kapitel beispielhaft vorgestellt. 


\section{Konkretisierung des Modells am Beispiel eines Service-Anliegens}

\subsection{Attribute und Kategorien von Service-Anliegen}

Incidents lassen sich wie beschrieben in die beiden Kategorien Service-Anliegen und Störungen unterteilen. Am Beispiel eines Service-Anliegens soll im Folgenden gezeigt werden, wie eine konkrete Implementierung des IncidentManagement-Prozesses auf der Grundlage der in Kapitel 3.2 vorgestellten SOA erfolgen kann.

Um die Anforderungen an die Implementierung zu definieren, sind in einem ersten Schritt die notwendigen Attribute eines Service-Anliegens und die wesentlichen Kategorien von Service-Anliegen zu definieren. Die ITIL sieht vor, dass ein Service-Anliegen die folgenden Attribute besitzt:

- Eindeutige Referenznummer

- Kategorie (Hauptkategorie und Unterkategorie)

- Datum/Zeit der Erfassung des Service-Anliegens

- Name, Abteilung, Telefonnummer oder Lokation des Nutzers

- Name oder ID des Bearbeiters im Service-Desk

- Rückrufmethode (z. B. Telefon oder E-Mail)

- Beschreibung des Service-Anliegens

- Priorität/Dringlichkeitsstufe

- Status des Service-Anliegens (z. B. aktiv, in Bearbeitung, abgeschlossen)

- Betroffene Configuration-Items in der Configuration-Management-Database

- Datum/Zeit des Abschlusses des Service-Anliegens

Die Daten werden in der Praxis entweder durch das System automatisch generiert oder durch einen Service-Desk-Mitarbeiter erfasst. Dazu sind Angaben seitens des Nutzers erforderlich, bspw. zur Identität des Nutzers, zur gewünschten Rückrufmethode und zur Art des Service-Anliegens. Teilweise unterstützen die eingesetzten Systeme diesen Prozess, etwa indem Nutzer anhand ihrer Telefonnummer automatisch identifziert und die nutzerspezifischen Daten aus einer Datenbank extrahiert und zugeordnet werden.

Eine Analyse der sechs betrachteten Unternehmen ergab die folgenden typischen Kategorien von Service-Anliegen:

- Beschaffung von Hardware 
- Beschaffung von Software

- Installation von Hardware

- Einrichtung von Hardware (z. B. PC oder Drucker)

- Installation von Software

- Einrichtung von Software

- Erteilung von Zugriffsberechtigungen (z. B. auf Netzwerkordner oder Anwendungen) und Passwörtern

- Einrichtung von E-Mail-Accounts

- Namens- und Passwortänderungen

- Durchführung von Schulungsmassnahmen

- Erteilung genereller Auskünfte

In der Regel existieren auch übergeordnete Kategorien, wie z. B. ein ServiceAnliegen des Typs "Eintritt neuer Mitarbeiter". Dieses setzt sich aus mehreren Einzel-Services zusammen, etwa der Beschaffung, Installation und Einrichtung von Hard- und Software, der Einrichtung eines E-Mail-Accounts und der Durchführung von Schulungsmassnahmen.

\subsection{Service-Request-Management auf Basis von WSDL und BPEL4WS}

Aufbauend auf den in Kapitel 4.1 beschriebenen Kategorien und Attributen von Incidents des ITIL-Referenzmodels wird in diesem Kapitel detailliert dargestellt, wie der aggregierte Web-Service Service-Request-Management-Service mit Hilfe der WSDL und BPEL4WS implementiert werden kann. Am Beispiel der EinzelServices "Beschaffung Hardware", "Installation Hardware" und "Konfiguration EMail-Account" kann das Service-Anliegen „Eintritt neuer Mitarbeiter“ beispielhaft zusammengefasst werden. Abbildung 6 stellt eine exemplarische Implementierung im Detail dar. 


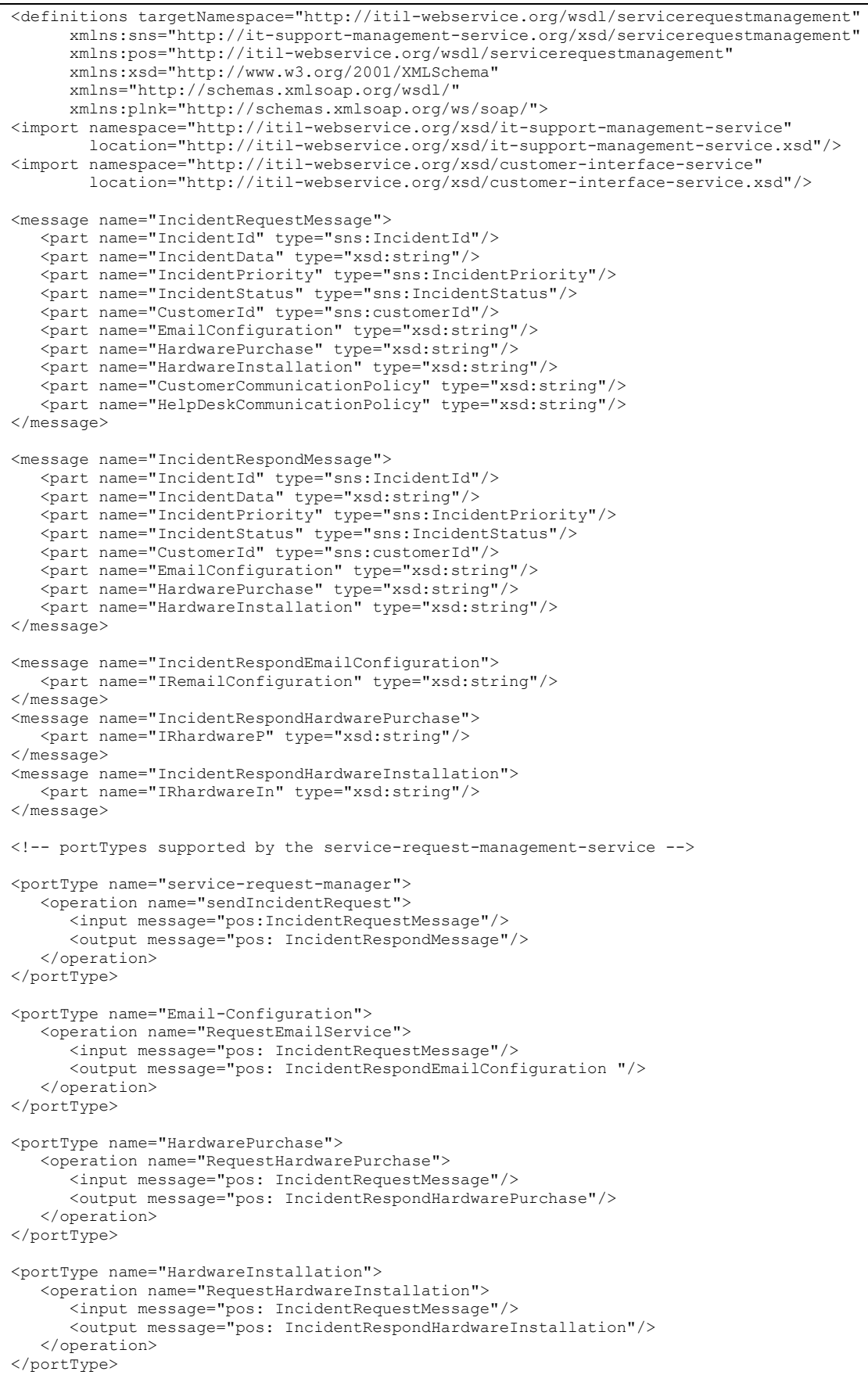

Abbildung 6: Implementierung des Service-Anliegens "Eintritt neuer Mitarbeiter" 
Innerhalb des WSDL-Dokumentes werden zunächst vorhandene Definitionen berücksichtigt, indem z. B. Schemata für XML, WSDL und SOAP integriert werden. Eine Berücksichtigung von Namenskonventionen und spezifischen Datentypen für das ITIL-Referenzmodell kann über die Importfunktion realisiert werden. Die Definition der Input- und Output-Nachrichtentypen und der jeweils zu verwendenden Attribute innerhalb des aggregierten Web-Services „Service-RequestManagement“ erfolgt im nächsten Teil des Dokumentes. Die „IncidentRequestMessage“ wird von dem Web-Service „Service-Request-Manager“ über die Transaktion „Request - multiple Responds“ an alle verfügbaren Web-Services innerhalb des aggregierten Web-Services "Service-Request-Management-Service" versendet. Die einzelnen atomaren Web-Services, z. B. „E-Mail-Configuration“, „Hardware Purchase“ und „Hardware Installation“, nutzen dann die Nachrichtentypen „IncidentRespondEmailConfiguration“, „IncidentRespondHardwarePurcha$\mathrm{se}^{\text {“ }}$ und „IncidentRespondHardwareInstallation“, um auf die Anfrage des WebServices „Service-Request-Manager“ zu antworten. Hierbei werden die verfügbaren Qualitäten und Ressourcen an den „Service-Request-Manager“ übertragen. Dieser wählt passende Web-Services mit Hilfe geeigneter Matchingfunktionen aus und bündelt diese zu einem aggregierten Web-Service. Die spezifische Ausprägung des aggregierten Web-Services „Eintritt neuer Mitarbeiter“ wird dann als Nachrichtentyp „IncidentRespondMessage“ vom Web Service „Service-RequestManager" an den aggregierten Web-Service "Service-Request-ManagementService" versendet. Dort kann z. B. eine abschließende Qualitätssicherung mit Hilfe einer geeigneten Funktion erfolgen, bevor die Nachricht an den Web-Service „Incident abschließen“ versendet wird. Mit Hilfe der Nachrichtenattribute „HelpDeskCommunicationPolicy“ und „CustomerCommunicationPolicy“ kann schliesslich vorab die Kommunikationsart mit dem Service-Nachfrager und dem HelpDesk-Mitarbeiter festgelegt werden.

\section{Praktische Erkenntnisse}

Aus den Fallstudien konnten eine Reihe praktischer Erkenntnisse gewonnen werden. Interessant sind in diesem Zusammenhang vor allem Erkenntnisse zu den Nutzeneffekten und kritischen Erfolgsfaktoren.

Der Nutzen eines ITIL-konformen IT-Service-Support ist aus Sicht der betrachteten Unternehmen vor allem in drei Punkten begründet:

- Es konnte eine Steigerung der Kunden- und Serviceorientierung des ITDienstleisters erreicht werden. Störungen, Fehler und Probleme wurden schneller behoben und Service-Anliegen zügiger bearbeitet. Die Kunden- bzw. Anwenderzufriedenheit ließ sich nachweislich verbessern. 
- Die Effizienz der IT-Leistungserstellung nahm zu. Grund hierfür waren vor allem Standardisierungsbemühungen und Prozessoptimierungen. So konnten beispielsweise die Support-Prozesse konsequent an den ITIL "Best Practices" ausgerichtet werden.

- Die Transparenz der Service-Support-Prozesse erhöhte sich aus Sicht der Kunden. Eine genaue Leistungsdefinition und der Einsatz von Kennzahlensystemen ermöglichte eine service-orientierte Ausgestaltung der Leistungsbeziehung zwischen Kunde und IT-Dienstleister.

Die Motivation zur Nutzung von Web-Services für die Umsetzung eines ITILkonformen IT-Service-Support liegt vor allem in der Steigerung der Interoperabilitäts- und Integrationsfähigkeit zwischenbetrieblicher und innerbetrieblicher Geschäftsprozesse auf der Grundlage standardisierter Technologien. Die dynamische Aggregation (Kopplung) und der Datenaustausch von Web-Services kann, z. B. mit Hilfe der Web-Services Choreography Description Language (WS-CDL), gesteuert und überwacht werden. Web-Services können in öffentlichen und privaten Verzeichnissen (z. B. UDDI, WS-Discovery) strukturiert beschrieben, gesucht und bewertet werden. Schließlich werden Sicherheit, Vertrauen und Privatsphäre durch die eingesetzten Standards für Web-Services gefördert.

Durchgeführte Praxisprojekte haben gezeigt, dass die nur zögerliche Akzeptanz bei der Nutzung von webbasierten Diensten nicht in erster Linie technologisch begründet ist, sondern primär auf Wissens- und Vertrauensdefiziten der Anbieter und Nachfrager von webbasierten Diensten beruht. Kataloge für webbasierte Dienste (z. B. asperado ${ }^{2}$ ) können zur Überwindung bestehender Kommunikationsund Vertrauensdefizite eingesetzt werden. Durch eine transparente Darstellung und Kommunikation der angebotenen webbasierten Dienste werden bestehende Informationsasymmetrien reduziert.

Die folgenden Maßnahmen erwiesen sich in den betrachteten Unternehmen als kritisch für den Projekterfolg:

- Aufzeigen kurzfristiger Erfolge (Quick-Wins), die durch ITIL-konforme ITService-Support-Prozesse erzielt werden können.

- Transformation vom projekt- zum produktorientierten Ansatz.

- Kontinuierliche Verbesserungen anstreben.

- Internes Marketing zur Schaffung von Akzeptanz und Verständnis.

- Top-Management-Support.

- Flächendeckende ITIL-Schulungen durchführen.

2 http://www.asperado.com 


\section{Zusammenfassung und Ausblick}

Der vorliegende Beitrag stellt auf der Basis des ITIL-Referenzmodells einen generischen Prozess für das Incident-Management vor und zeigt beispielhaft eine technische Implementierung auf der Grundlage einer Service-orientierten-Architektur. Der generische Incident-Management-Prozess wurde aus sechs Fallstudien abgeleitet. Für die Implementierung wurden atomare und aggregrierte Web-Services definiert und die Interaktion zwischen den Web-Services über Transaktionen abgewickelt. Am Beispiel eines Service-Anliegens wurde konkret gezeigt, wie die einzelnen Elemente eines XML-Elementes eines ITIL-konformen Web-Services ausgeprägt sind.

Für eine vollständige Implementierung sind die im Modell enthaltenen WebServices zu spezifizieren und mit Funktionalität zu füllen. Darüber hinaus müssen die Interaktionsprozesse zwischen den Web-Services eindeutig definiert werden.

\section{Literatur}

[BöKr04] Böhmann, T.; Krcmar, H.: Grundlagen und Entwicklungstrends im ITServicemanagement. In: HMD - Praxis der Wirtschaftsinformatik, 41(2004)237, S. 721

[BPEL03] Specification: Business Process Execution Language for Web Services Version 1.1. IBM developerWorks, http://www-106.ibm.com/developerworks/library/WS bpel, Aufruf am 12. Juli 2004

[Giso01] Gisolfi, D.: Is Web services the reincarnation of CORBA? IBM developer Works, 1. Juli 2001, http://www-106.ibm.com/developerworks/webservices/library/WS arc3/, Aufruf am 12 Juli 2004

[He03] He, H.: What is Service-Oriented Architecture? O'REILLY webservices.xml.com, September 2003, http://webservices.xml.com/pub/a/ws/2003/09/30/soa.html, Aufruf am 12 Juli 2004

[HoHo98] Holst, H.; Holst, J.: IT-Produkt und Klientenmanagement. In: Information Management, Nr. 2, 1998, S. 56-65

[HoHu03] Hochstein, A.; Hunziker, A.: Service-orientierte Referenzmodelle des ITManagements. In: HMD - Praxis der Wirtschaftsinformatik, 40(2003)232, S. 45-56

[HoZa04] Hochstein, A.; Zarnekow, R.; Brenner, W.: ITIL als Common-PracticeReferenzmodell für das IT-Service-Management. In: Wirtschaftsinformatik, 46(2004)5

[ITSM04] IT Service Management Forum: What is the itSMF? http://www.itsmf.com/about/whatis.asp, Auruf am 12 Juli 2004 
[LeDi04] Lehner, F.; Dirmeyer, J.; Lindner, O.: Softwarewerkzeuge zum ITServivemanagement. In: HMD - Praxis der Wirtschaftsinformatik, 41(2004)237, S. 6779

[OASIS 2003] Organization for the Advancement of Structured Information Standards, UDDI Version 3.0.1., Committee Specification, 14. Oktober 2003, http://uddi.org/pubs/uddi-v3.0.1-20031014.htm, Aufruf am 12. Juli 2004

[OGC00] Office of Government Commerce: ITIL Best Practice for Service Support. The Stationary Office, Norwich, 2004

[QuWi03] Quantz, J., Wichmann T.: Basisreport Integration mit Web Services. Berlecon Research, August 2003

[Stal02] Stal, M.: Web Services - Beyond Component-Based Computing. In: Communications of the ACM, 45(2002)10, S. 71-76

[Stev02] Stevens, M.: The Benefits of a Service-Oriented Architecture. developer.com, April 2002, http://www.developer.com/tech/article.php/1041191, Aufruf am 12. Juli 2004

[TaWu03] Tamm, G., Wuensche M.: Strategies to reduce information asymmetry in web service market, Proceedings 11th European Conference on Information Systems (ECIS), Neapel, Italien, 2003

[W3C00] World Wide Web Consortium: Simple Object Access Protocol (SOAP) 1.1. W3C Note, 8. Mai 2000, http://www.w3.org/TR/soap, Aufruf am 12. Juli 2004

[W3C01] World Wide Web Consortium: Web Services Description Language (WSDL) 1.1. W3C Note, 28. März 2001, http://www.w3.org/TR/wsdl, Aufruf am 12. Juli 2004

[W3C03] World Wide Web Consortium: SOAP Version 1.2 Part 1: Messaging Framework, 24. Juni 2003, http://www.w3.org/TR/soap12-part1, Aufruf am 12. Juli 2004

[Zarn04] Zarnekow, R.: Produktorientiertes Informationsmanagement. In: Zarnekow, R.; Brenner, W.; Grohmann, H. H. (Hrsg.) Informationsmanagement - Konzepte und Strategien für die Praxis. Dpunkt, Heidelberg, 2004, S. 41-56 